\title{
A Japanese Adult Case of Forme Fruste Cystic Fibrosis
}

Hiroaki Oka, Hiroshi Ishii, Kenji Kishi, Atsushi Yokoyama, Kosaku Komiya, Satoshi Otani, Daisuke Yoshioka, Ryo Shirai, Issei Tokimatsu, Kazufumi Hiramatsu and Jun-ichi Kadota

Key words: cystic fibrosis, CFTR mutation

(Inter Med 49: 1251-1252, 2010)

(DOI: 10.2169/internalmedicine.49.3407)
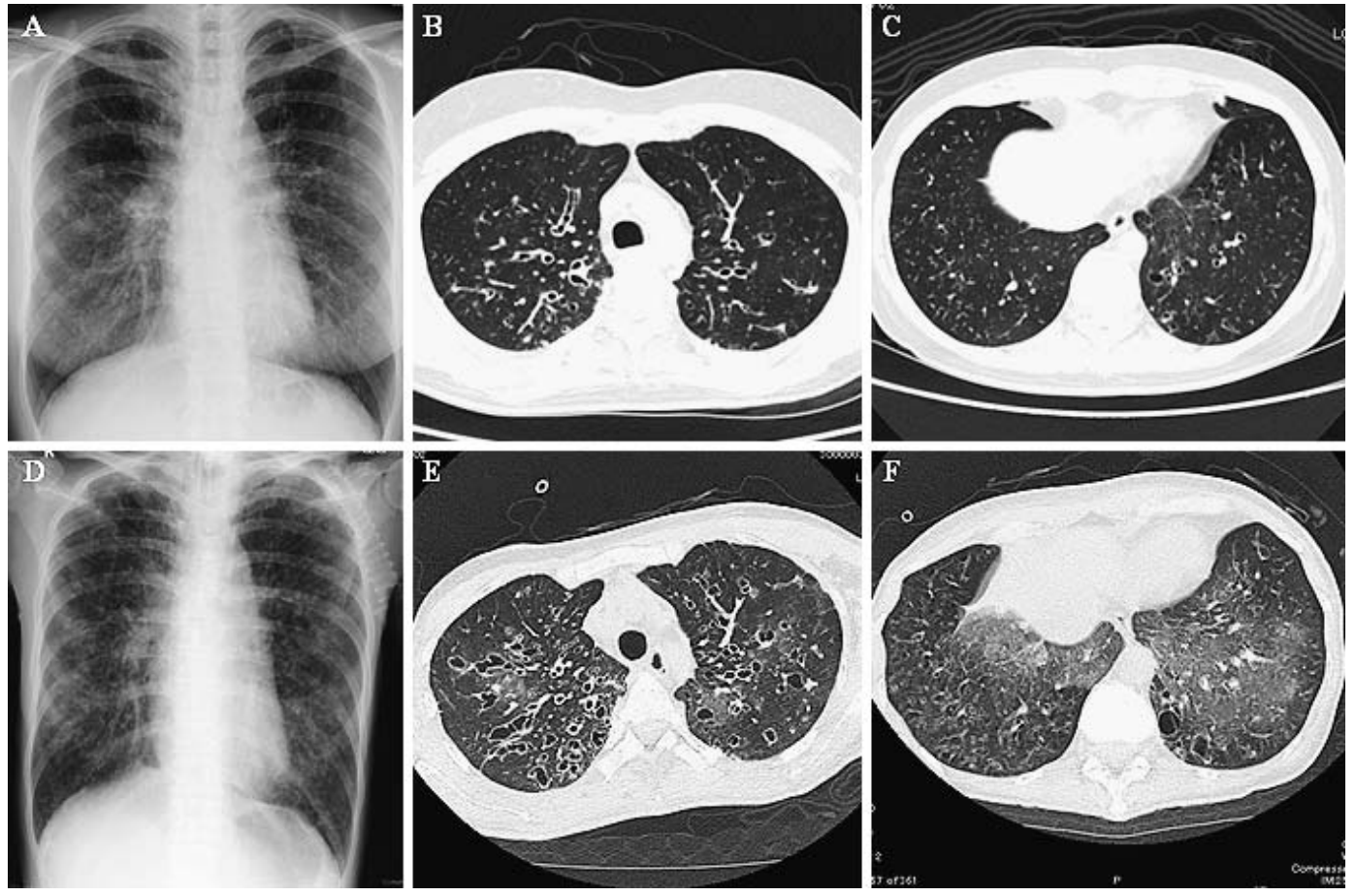

Picture 1. (A) A chest roentgenogram in 2003 showed scattered small nodular shadows in both lung fields. (B, C) Computed tomography (CT) of the chest in 2003 showed bronchial wall thickening and centrilobular nodules predominantly in both upper lobes. (D-F) A chest roentgenogram and CT in 2009 showed exacerbation bilaterally with marked bronchiectasis.

A 43-year-old woman was admitted to undergo surgical treatment for right pneumothorax in 2008. She had no noteworthy family history. She was married (non-intermarriage) but did not have any children. Although she had been treated with macrolides for diffuse panbronchiolitis (DPB) with chronic sinusitis since 2003, her disease condition had been progressive (Picture 1). Unlike DPB, Staphylococcus aureus had been mainly detected in her sputum and the chest roentgenogram and computed tomography revealed bronchiectasis predominantly in the upper lobes (Picture 1). The resected lung specimen did not pathologically confirm
DPB. Although the sweat chloride test had not been done, she was diagnosed to have forme fruste cystic fibrosis $(\mathrm{CF})$ based on a gene sequence analysis (Ambry Genetics Corporation, Aliso Viejo, $\mathrm{CA}$ ) of the $\mathrm{CF}$ transmembrane conductance regulator $(C F T R)$. Unfortunately, we have not been able to obtain permission from her family or relatives for genetic testing. During the course of disease, she was free of any significant extrapulmonary involvement, such as either pancreatic exocrine insufficiency or meconium ileus. Lung transplantation was considered, however, she eventually died of respiratory failure with a chronic Pseudomonas 
aeruginosa infection in 2009. CF is rare in Japan, and this is only the second case which carried both E217G and Q $1352 \mathrm{H}$ mutations in the CFTR gene $(1,2)$.

\section{Acknowledgement}

We thank Dr. K. Yoshimura (Respiratory Center, Toranomon Hospital, Tokyo) for valuable assistance in the diagnosis.

\section{References}

1. Yamashiro $Y$, Shimizu T, Oguchi $S$, Shioya $T$, Nagata $S$, Ohtsuka Y. The estimated incidence of cystic fibrosis in Japan. J Pediatr Gastroenterol Nutr 24: 544-547, 1997.

2. Yoshimura K, Anzai C. Cystic fibrosis in the Japanese population. Nihon Kyobu Rinsyo 68: 693-705, 2009 (in Japanese).

\footnotetext{
(C) 2010 The Japanese Society of Internal Medicine http://www.naika.or.jp/imindex.html
} 